\title{
MINLP Optimization Algorithm for the Synthesis of Heat and Work Exchange Networks
}

\author{
Viviani C. Onishi ${ }^{\text {a,*, }}$, Mauro A. S. S. Ravagnani, José A. Caballero \\ ${ }^{a}$ Department of Chemical Engineering, State University of Maringá, Av. Colombo 5790, \\ Maringá-PR 87020-900, Brazil
}

\begin{abstract}
This paper introduces a new optimization model for the simultaneous synthesis of heat and work exchange networks. The work integration is performed in the work exchange network (WEN), while the heat integration is carried out in the heat exchanger network (HEN). In the WEN synthesis, streams at high-pressure (HP) and low-pressure (LP) are subjected to pressure manipulation stages, via turbines and compressors running on common shafts and stand-alone equipment. The model allows the use of several units of single-shaft-turbine-compressor (SSTC), as well as helper motors and generators to respond to any shortage and/or excess of energy, respectively, in the SSTC axes. The heat integration of the streams occurs in the HEN between each WEN stage. Thus, as the inlet and outlet streams temperatures in the HEN are dependent of the WEN design, they must be considered as optimization variables. The proposed multi-stage superstructure is formulated in mixed-integer nonlinear programming (MINLP), in order to minimize the total annualized cost composed by capital and operational expenses. A case study is conducted to verify the accuracy of the proposed approach. The results indicate that the heat integration between the WEN stages is essential to enhance the work integration, and to reduce the total cost of process due the need of a smaller amount of hot and cold utilities.
\end{abstract}

Keywords: optimization; mixed-integer nonlinear programming (MINLP); work exchange network (WEN); heat exchanger network (HEN); heat integration.

\section{Introduction}

Heat and work are two forms of energy frequently available in processing plants. The optimization of energy integration through the application of innovative strategies, and development of more efficient processing techniques is the most effective way to reduce process costs, and minimize environmental impacts related to high energy consumption (Lara et al., 2013). The pressure manipulation of streams is extremely important in many industrial processes, such as production of ammonia and natural gas, wherein is responsible for the consumption of large energy amounts and, consequently, high operating expenses. Although the synthesis of heat exchanger networks (HENs) has been intensively studied since the energy crisis in the 1970s (Ravagnani and Silva, 2012), with significant impact on the industry (Huang and Karimi, 2013), the literature about the problem of work integration is rather limited (Onishi et al., 2013).

Huang and Fan (1996) introduced the work exchange network (WEN) problem, defining operational principles for work exchange among two process streams. Aspelund et al. (2007) presented a method based on Extended Pinch Analysis and Design (ExPAnD) to minimize energy requirements of the system. In their work, compressors and turbines are used separately, with any mention to the use of pressure manipulation equipment operating in a common axis. Moreover, only the aspects related 
to the exergy analysis were evaluated. Razib et al. (2012) presented a preliminary model for WENs synthesis, with the goal of minimizing the total cost of the network. The authors proposed a superstructure to optimize the pressure recovery of streams, via equipment running in a single-shaft-turbine-compressor (SSTC). Nevertheless, the model allows the use of only one SSTC axis unit restricted to a fixed rotational speed. In addition, the heat integration is not considered and the cooling and heating of streams is performed by heaters and coolers located in the end of each WEN stage.

A new optimization model is proposed for the simultaneous synthesis of WEN with heat integration of streams. In the WEN design, streams at high-pressure (HP) and lowpressure (LP) exchange work in pressure manipulation stages, via turbines and compressors running on common shafts and stand-alone equipment. The model allows the use of several SSTC axes units operating at any rotational speed. Generators and helper motors are used, respectively, to convert work excess into electricity and to fill for power shortage on the SSTC axes. Between the compression and expansion stages of the WEN, the streams are sent to the HEN to promote heat integration. The pressure and heat recovery of streams are performed in a multi-stage superstructure, formulated as a mixed-integer nonlinear programming (MINLP) problem, in order to minimize the total annualized cost. A case study is conducted to verify the accuracy of the proposed model. The results indicate that the heat integration between WEN stages is essential to enhance the work integration, and to reduce the total cost of the network.

\section{Problem statement}

Given a set of HP and LP gaseous streams with known mass flows, heat capacities, supply state (inlet pressure and temperature) and target state (outlet pressure and temperature); besides electricity, thermal utilities for heating and cooling, equipment for pressure manipulation and heat transfer, and their respective costs. The main objective is to synthesize an optimal WEN by minimizing the total annualized cost composed by the operational expenses-including the revenue from electricity generation-and capital cost of investment in the various units of the network. The leading idea of the WEN synthesis is to promote the work exchange between HP and LP streams, via turbines and compressors running on a common SSTC axis. The SSTC axis allows the pressure recovery among different streams in several expansion and compression stages through single-stage and parallel equipment, as well as the use of helper motors and generators to respond to any shortage and/or excess of energy, respectively. Moreover, stand-alone compressors, turbines and valves are also used to compose the WEN in addition to several SSTC axes units. The HEN synthesis is performed simultaneously to the WEN design, by recovering heat from process streams aiming to improve the pressure recovery. Thus, while the HEN only involves the heat integration of streams, the WEN involves the work integration or work and heat integration, using SSTC equipment, stand-alone turbines and compressors, valves, helper motors and generators and, when necessary, heat exchangers, heaters and coolers.

\section{MINLP optimization model}

In this paper, the proposed model for the WEN synthesis with heat integration is developed in a MINLP formulation. The proposed WEN superstructure is composed by $n$ stages of compression or expansion for each stream. Consequently, each HP stream goes through $n$ expansion stages and, analogously, each LP stream undergoes $n$ compression stages. The pressure recovery is performed by single-stage and/or parallel 
equipment—namely compressors and turbines - associated to a common shaft (SSTC). Several units of SSTC axes can be used. However, the global energy balance should be respected in each SSTC axis unit, i.e., the expansion work must be equal to the compression work:

$W g_{e}+\sum_{i=1}^{L P} \sum_{n=1}^{N} \sum_{k=1}^{K} W e_{i, n, e, k}=W m_{e}+\sum_{j=1}^{H P} \sum_{n=1}^{N} \sum_{k=1}^{K} W e_{j, n, e, k} \quad i \in L P, j \in H P, n \in N, k \in K, e \in E$

For this reason, helper motors and generators (turbines) allocated on the SSTC axes, and stand-alone compressors, turbines and valves are also used in the network. At each compression and expansion stage, the overall mass flow is splitted between various flows, through the pressure manipulation equipment that compose each of these stages. In the end of each stage, the sub-streams pass through a mixer, reforming the parent streams. Although the outlet pressure is the same in all equipment of each expansion stage, the temperature in the outlet of each device is different. Thus, energy and mass balances should be performed at the mixing points.

Besides the work integration, the HP and LP streams are submitted to simultaneous heat integration in the HEN. As a result, the inlet temperatures in the HEN should be connected to the outlet streams temperatures from the WEN stages. The formulation is based on the model for HEN design proposed by Yee and Grossmann (1990), wherein streams splits is considered, and isothermal mixing and constant heat transfer coefficients are assumed. However, the model is extended to include the unknown intermediate temperatures from the WEN, which are treated as optimization variables. The HEN superstructure is divided in stages according to temperature at mixing point, in which all hot streams can exchange heat with all cold streams. Thus, the HP streams are considered as cold streams and the LP streams as hot streams, since high temperatures favor the expansion process, as well low temperatures improve the compression process. Heaters and coolers are placed on the end of the streams, in order to guarantee that their target temperature can be achieved. The objective function is composed by capital cost associated to the various units of the network and operational expenses.

The resulting model is highly complex, due the need to simultaneously optimize all parameters relating to WEN and HEN design. For simplification, the same number of stages is considered in both WEN and HEN, and the ideal gas model is used to describe the thermodynamic behavior of real gases. However, an isentropic efficiency factor is considered to adjust inevitable losses of efficiency of real processes. The proposed MINLP model is optimized with the DICOPT solver under GAMS software (version 24.02). The high non-convexity and nonlinearity of the mathematical formulation does not guarantee the global optimum. However, some numerical experiments with this model showed that increasing the number of major iterations, and solving feasibility problems that may arise from NLP, allow obtaining good solutions. For the solution of the model is crucial to adequately establish all limits of the variables.

\section{Case study}

An example is studied to verify the applicability of the proposed approach for the simultaneous synthesis of heat and work exchange networks. In this case study, the WEN is designed allowing work integration between two streams at high-pressure (HP1 and HP2), and two streams at low-pressure (LP1 and LP2).The goal is to obtain a 
network that presents minimum total annualized cost through the optimal energy integration. The maximum number of four compression stages and four expansion stages of the streams are considered for the WEN synthesis. The process streams are sent to the HEN to promote heat recovery between the stages of compression and expansion. Thus, the high-pressure streams HP1 and HP2 are considered as cold streams, and the low-pressure streams LP1 and LP2 are regarded as hot streams for the HEN design. The stream HP1 originates the cold streams C1 to C5 and the stream HP2 gives rise to cold streams C6 to C10. Similarly, the stream LP1 originates the hot streams $\mathrm{H} 1$ to $\mathrm{H} 5$ and the stream LP2 gives rise to hot streams H6 to H10. For the HEN synthesis, a superstructure with four stages of heat exchange and the possibility of streams splits are considered, and isothermal mixing is assumed. The flow rates $(F)$ and heat capacities $(C p)$ of all the streams are known constants. The problem data are presented in Table 1. The hot and cold utilities are available on $500 \mathrm{~K}$ and $288 \mathrm{~K}$, respectively. In addition, all unknown intermediate temperatures of streams are restricted between $288 \mathrm{~K}$ and $600 \mathrm{~K}$, and $f=0.18, \Delta T_{\min }=5 \mathrm{~K}, \eta=0.7, \mu=1.961 \mathrm{e}-3$ $\mathrm{K} / \mathrm{kPa}, R C_{\max }=3$ are also regarded.

In this case study, the use of two SSTC axes units is analyzed. The optimal WEN obtained is composed by two valves, two stand-alone turbines, four stand-alone compressors, four SSTC turbines, and three SSTC compressors. The stream HP1 is subjected to three expansion stages through two parallel SSTC turbines $(1,480.1 \mathrm{~kW}$ and $100 \mathrm{~kW}$ ) associated to the shaft 2, one valve, and one stand-alone turbine (848.4 $\mathrm{kW})$. The stream HP2 undergoes four expansion stages through one valve, two singlestage SSTC turbines (950 kW and $100 \mathrm{~kW})$ located on shaft 1 and shaft 2, respectively, and one stand-alone turbine $(698.3 \mathrm{~kW})$. Both streams LP1 and LP2 undergo three compression stages. The stream LP1 passes through two stand-alone compressors (282 $\mathrm{kW}$ and $950 \mathrm{~kW})$, and one single-stage SSTC compressor $(950 \mathrm{~kW})$ located on the shaft 1. The stream LP2 passes through one stand-alone compressor $(950 \mathrm{~kW})$, two parallel SSTC compressors $(950 \mathrm{~kW}$ and $730.1 \mathrm{~kW})$ associated to the shaft 2, and one standalone compressor $(950 \mathrm{~kW})$. The total work of expansion (compression) performed (consumed) by the SSTC turbines (SSTC compressors) is equal to $950 \mathrm{~kW}$ for the shaft 1 , and $1,680.1 \mathrm{~kW}$ for the shaft 2 . In the HEN design, four heat exchangers with heat exchanger areas $(A)$ of $390.5 \mathrm{~m}^{2}(Q=603.6 \mathrm{~kW}), 432.7 \mathrm{~m}^{2}(Q=742.1 \mathrm{~kW}), 581.3 \mathrm{~m}^{2}$ $(Q=1,083.7 \mathrm{~kW})$ and $918.2 \mathrm{~m}^{2}(Q=1,748.6 \mathrm{~kW})$ are needed. Moreover, two coolers $(A$ $=424.1 \mathrm{~m}^{2}$ with $Q=836.2 \mathrm{~kW}$, and $A=462.7 \mathrm{~m}^{2}$ with $Q=747.9 \mathrm{~kW}$ ) are used for the heat recovery. Figure 1 shows the optimal WEN configuration with heat integration obtained for this example. In this case, as there are no remains or lacks of energy in the shafts, no helper motor or generator is needed to satisfy the energy balance. However, two stand-alone turbines are used in the network configuration. Consequently, 1,546.7 $\mathrm{kW}$ of energy are produced in the WEN, and can be used in other stages of the process and/or for sale of electricity. The total annualized cost estimated is $6,279 \mathrm{k} \mathrm{US \$ \textrm {y } ^ { - 1 }}$, in

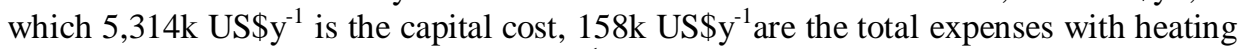

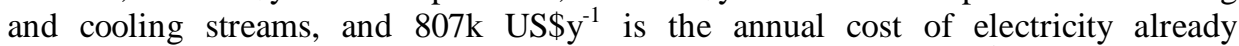
discounted the revenue generated by stand-alone turbines $\left(619 \mathrm{k} \mathrm{US} \$ \mathrm{y}^{-1}\right)$.

Table 1. Streams data for the example.

\begin{tabular}{cccccccc}
\hline Streams & $\begin{array}{c}F \\
(\mathrm{~kg} / \mathrm{s})\end{array}$ & $\begin{array}{c}C p \\
(\mathrm{~kJ} / \mathrm{kg} \mathrm{K})\end{array}$ & $\begin{array}{c}h \\
\left(\mathrm{~kW} / \mathrm{m}^{2} \mathrm{~K}\right)\end{array}$ & $\begin{array}{c}T_{I N} \\
(\mathrm{~K})\end{array}$ & $\begin{array}{c}T_{\text {OUT }} \\
(\mathrm{K})\end{array}$ & $\begin{array}{c}P_{\text {IN }} \\
(\mathrm{kPa})\end{array}$ & $\begin{array}{c}P_{\text {OUT }} \\
(\mathrm{kPa})\end{array}$ \\
\hline HP1 & 15 & 1.432 & 0.1 & 380 & 380 & 850 & 100 \\
HP2 & 18 & 0.982 & 0.1 & 400 & 400 & 980 & 180 \\
LP1 & 15 & 1.432 & 0.1 & 400 & 400 & 100 & 520 \\
\hline
\end{tabular}




\begin{tabular}{cccccccc}
\hline LP2 & 18 & 1.432 & 0.1 & 400 & 400 & 100 & 850 \\
\hline Cost data: $C E=455.04 \mathrm{US}^{-1} \mathrm{~kW} ; C V=400$ & $\mathrm{USSy}^{-1} \mathrm{~kW} ; C S=337{\mathrm{US} \$ \mathrm{y}^{-1} \mathrm{~kW} ; C W=100}^{\mathrm{USS} \$ \mathrm{y}^{-1} \mathrm{~kW}}$
\end{tabular}

In this example, if the network is synthetized with only one SSTC axis, the same equipment obtained with two axes is needed for the WEN design with heat integration. Clearly, as the networks obtained in both cases (i.e., with one and two SSTC axes) differ only in the number of SSTC axes used; both have the same total cost. Moreover, if the use of three SSTC axes units is forced, the total annualized cost increases that represent a sub-optimal solution. The mathematical model contains 3,900 continuous variables, 232 binary variables and 5,105 constraints with 17,710 Jacobian elements (non-zeros), in which 2,482 are nonlinear. The CPU time is $6.46 \mathrm{~min}$ and the optimal solution is found in the sixth major iteration. The example was solved using a personal computer with an Intel Core 2 Duo $2.40 \mathrm{GHz}$ processor and 3.00 GB RAM under Windows 7 Ultimate.

\section{Conclusions}

A new optimization model is proposed for the simultaneous synthesis of WEN with heat integration concomitant in the HEN. The multi-stage superstructure is developed in a MINLP formulation, aiming the optimal integration between heat and work of LP and HP streams, by minimizing the total annualized cost of the network.

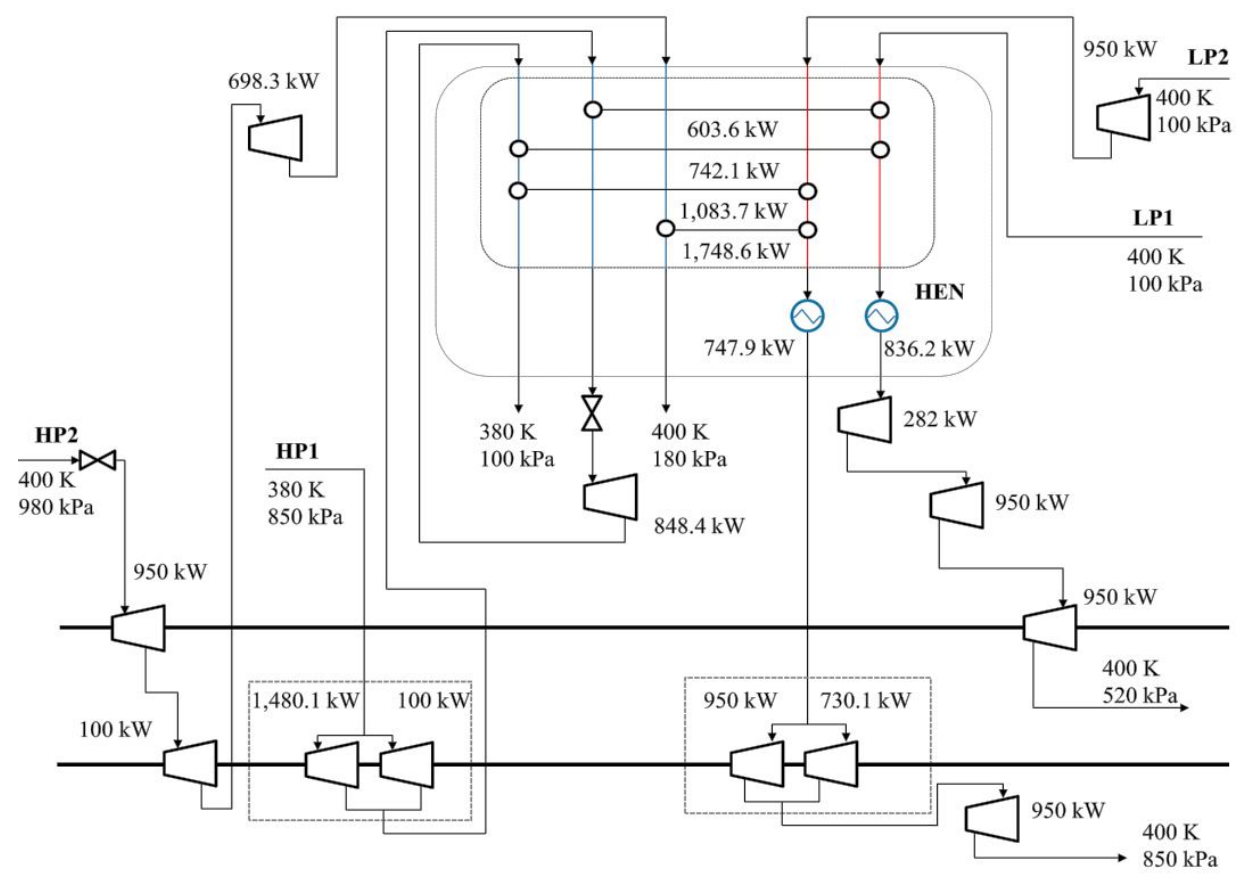

Figure 1. Optimal configuration of the WEN with heat integration obtained for the case study.

The results obtained indicate that the heat integration between the WEN stages of expansion and compression is essential to enhance the work integration. Furthermore, the heat recovery from streams reduces the total annualized cost in $29 \%$ as a consequence of the decrease of the operational expenses related to the use of thermal utilities. The total cost is also reduced in $10 \%$ due the revenue generated from the 
expansion work produced by the stand-alone turbines. In the case study, the same WEN with heat integration can be designed with the use of one or two SSTC axes units. Thus, besides the economic factor, the space available in the processing plant should be considered to choose the best WEN design. The larger number of equipment running on the same SSTC unit, the larger the difficulty to keep them under the same operating conditions will be, since all must be kept under the same rotational speed. In contrast, the use of a larger number of shafts may request a bigger space in the process plant.

\section{Nomenclature}

\begin{tabular}{|c|c|c|c|}
\hline$C E$ & cost parameter for electricity & $W e$ & SSTC compressor/turbine work \\
\hline$C R_{\max }$ & maximum compression ratio & $W g$ & generator work \\
\hline$C S$ & cost parameter for the heating & $W m$ & helper motor work \\
\hline$C V$ & cost parameter for the electric & $\eta$ & isentropic efficiency \\
\hline & power revenue & $\mu$ & Joule-Thompson coefficient \\
\hline$C W$ & cost parameter for the cooling & \multicolumn{2}{|c|}{ Subscripts } \\
\hline$f$ & annualization factor for the cost & $e$ & SSTC axes units \\
\hline$P$ & pressure & $i$ & LP streams (hot) \\
\hline$T$ & temperature & $j$ & HP streams (cold) \\
\hline \multirow[t]{2}{*}{$\Delta T_{\min }$} & minimal approximation & $k$ & streams splits \\
\hline & temperature & $n$ & stages in the WEN \\
\hline
\end{tabular}

\section{Acknowledgements}

The financial supports by the Brazilian agency "Coordenação de Aperfeiçoamento de Pessoal de Nível Superior - CAPES" (process n ${ }^{\circ} 10758 / 12-7$ ), and the Spanish Ministry of Science and Innovation and Ministry of Economy and Competitiveness (under project CTQ2012-37039-C02-02) are gratefully acknowledged.

Affiliation of José A. Caballero is Department of Chemical Engineering, University of Alicante, Ap Correos 99, Alicante03080, Spain.

Viviani C. Onishi also has the following affiliation: CAPES Foundation, Ministry of Education of Brazil, Brasília-DF 7004020, Brazil

pg51551@uem.br/viviani.onishi@hotmail.com

\section{References}

A. Aspelund, D.O. Berstad, T. Gundersen, 2007, An extended pinch analysis and design procedure utilizing pressure based exergy for subambient cooling, Applied Thermal Engineering, 27, 2633-2649.

K.F. Huang, I.A. Karimi, 2013, Simultaneous synthesis approaches for cost-effective heat exchanger networks, Chemical Engineering Science, 98, 231-245.

M.A.A.S. Ravagnani, A.P. Silva, 2012, Retrofit of heat exchanger networks including the detailed equipment design, Computer Aided Chemical Engineering, 31, 235-239.

M.S. Razib, M.M.F. Hasan, I.A. Karimi, 2012, Preliminary synthesis of work exchange networks, Computers and Chemical Engineering, 37, 262-277.

T.F. Yee, I.E. Grossmann, 1990, Simultaneous optimization models for heat integration - II. Heat exchanger network synthesis, Computers and Chemical Engineering, 14, 10, 1165-1184.

V.C. Onishi, M.A.S.S. Ravagnani, J.A. Caballero, 2013, Simultaneous synthesis of heat exchanger networks with pressure recovery: Optimal integration between heat and work. AIChE Journal, doi:10.1002/aic.14314.

Y. Lara, P. Lisbona, A. Martínez, L.M. Romeo, 2013, Design and analysis of heat exchanger networks for integrated Ca-looping systems, Applied Energy, 111, 690-700.

Y.L. Huang, L.T. Fan, 1996, Analysis of a work exchanger network, Industrial and Engineering Chemistry Research, 35, 3528-3538. 